\title{
Estado actual de investigación en parálisis cerebral y envejecimiento: revisión sistemática
}

\section{Current state of research in cerebral palsy and aging: systematic review}

\begin{abstract}
Resumen
El objetivo de la presente revisión es analizar las publicaciones en el área de parálisis cerebral y envejecimiento, respecto a tipo y volumen de publicaciones, metodología, áreas temáticas y edad de corte de la muestra. La importancia de la temática se deriva del incremento de la esperanza de vida de este colectivo y su aumento demográfico, que conlleva a que surja un nuevo colectivo con demandas específicas a las que la comunidad científica debe dar respuesta. Se plantea una búsqueda en las principales bases de datos y un análisis siguiendo el método PRISMA, con el fin de que la revisión sea sistemática y replicable. Tras el análisis de las publicaciones se destaca la predominancia de estudios empíricos del área médica, debilidades metodológicas comunes al área de investigación y la necesidad de realizar investigaciones longitudinales y de corte psicosocial que permitan dar respuesta de manera integral.
\end{abstract}

\section{Palabras clave}

Parálisis cerebral, envejecimiento, discapacidad, revisión sistemática.

\begin{abstract}
The aim of this paper review is to analyse the publications in the area of cerebral palsy and ageing, with respect to type and volume of publications, methodology, thematic areas and age of cut of the sample. The importance of the theme derives from the increase in the life expectancy of this group and its demographic growth, which leads to the emergence of a new group with specific demands to which the scientific community must respond. A search of the main databases and an analysis following the PRISMA method are proposed, in order to make the review systematic and replicable. After the analysis of the publications, the predominance of empirical studies in the medical area, methodological weaknesses common to the research area and the need to carry out longitudinal and psychosocial research that allows an integral response are highlighted.
\end{abstract}

\section{Keywords}

Cerebral palsy, ageing, disability, systematic review.

\section{Patricia Solís García \\ <patricia.solis@unir.net>}

Universidad Internacional de La Rioja. España

\section{Sara Real Castelao}

<sarreal@ponferrada.uned.es>

Centro Asociado UNED Ponferrada. España

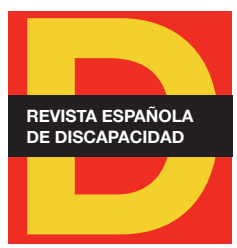

Para citar:

Solís, P. y Real, S. (2019): "Estado actual de investigación en parálisis cerebral y envejecimiento: revisión sistemática". Revista Española de Discapacidad, 7 (II): 103-122.

Doi: <https://doi.org/10.5569/23405104.07.02.06>

Fecha de recepción: 27-08-2018 Fecha de aceptación: 30-10-2019 


\section{Introducción}

En los países occidentales actualmente se está haciendo frente a un cambio demográfico que está modificando las estructuras sociales hasta ahora conocidas (Coyle y Mutchler, 2017), a causa del progresivo envejecimiento poblacional. Este cambio es de tal magnitud que algunos autores lo denominan como "revolución demográfica" o "transición demográfica" por su efecto en las políticas sociales (Petretto et al., 2019). Este cambio demográfico afecta también a las personas con discapacidad en general, así como a las personas con discapacidad intelectual ( $\mathrm{Ng}$ et al., 2015) y a colectivos como el de parálisis cerebral (PC), en particular. Estas personas deben afrontar un importante deterioro propio de un envejecimiento que no tiene precedentes históricos (Coyle et al., 2016). Colectivos como el de discapacidad intelectual y PC precisan diversos apoyos y servicios personalizados que respondan a las necesidades que se derivan de su envejecimiento (Moro et al., 2017; Schepens et al., 2017). Asimismo, el envejecimiento en este grupo se adelanta temporalmente y puede señalarse como motivo de los problemas secundarios que aparecen en estas personas con discapacidad (Alcedo et al. 2017; Martin et al., 2017).

Sin embargo, pese a encontrar gran volumen de comunicación científica que aborda la temática de la discapacidad, se aprecia un olvido hacia las necesidades específicas de aquellos que envejecen (Jahoda et al., 2015). Esta escasez de publicaciones es más patente en el caso de las personas con PC (Svien et al. 2008). Esta falta de datos específicos y difusión científica de las necesidades mantiene la situación de exclusión e invisibilización que presentan respecto a la población general y a otros colectivos de personas con discapacidad (Verdugo y Navas, 2017).

McCallion et al., (2019) señalan las dificultades para realizar un seguimiento de las personas de edad con discapacidad intelectual debido a las diferencias en las definiciones y la forma en que se recopilan los datos. Las investigaciones en la temática indican que las personas con discapacidad afrontan su envejecimiento de manera prematura (Carmeli et al., 2004), se halla una mayor prevalencia de problemas cardiovasculares, respiratorios, gastrointestinales, endocrinos y neurológicos que en población general (Berjano y García, 2010; Strydom et al., 2019) y se aprecia una disminución significativa de la participación y la interacción social (Judge et al., 2010). Por ello, se precisa un estudio de las demandas específicas de este colectivo ante el reto de dar respuesta a la aparición de nuevas necesidades como al agravamiento de las ya existentes (Beadle-Brown et al., 2015).

En el caso de la PC, gracias a los avances médicos más personas residentes en países desarrollados están sobreviviendo a la adolescencia y la edad adulta (Frisch y Msall, 2013). Este aumento de la esperanza de vida requiere que exista un avance en la investigación que permita a la sociedad estar en condiciones de mejorar la calidad de vida a medida que estas personas envejecen (Balandin et al., 2006). La PC presenta una prevalencia global entre 2 y 3 casos por cada 1000 habitantes, aunque en los países occidentales algunos estudios aportan menor incidencia (Robaina et al., 2007). En España, según la última encuesta realizada a toda la población en temática de discapacidad, la tasa se sitúa en el 1,95 (INE, 2008). Sin embargo, a pesar de ser un colectivo numeroso, ha sido una de las formas clínicas ligadas a la discapacidad más incomprendidas debido a las dificultades de estudio que presenta este trastorno y que hacen difícil hallar un consenso en cuanto a su definición y clasificación, que ha supuesto una limitación en las interpretaciones de los estudios epidemiológicos (Robaina et al., 2007). De este modo, hasta el año 2007 se ha considerado a la PC como 
"un término paraguas que engloba un grupo de síndromes con alteraciones motoras no progresivas, pero a menudo cambiantes, secundarias a lesiones $\mathrm{o}$ alteraciones del cerebro producidas en los primeros estadios de su desarrollo" (Pueyo-Benito y Vendrell-Gómez, 2002: 1080). El desarrollo en neuroimagen, neurobiología y patología asociada conllevó un cambio en el concepto, y, por extensión en la clasificación y comorbilidades asociadas de la PC, dando lugar a una definición fundamentada en la Clasificación Internacional de Funcionamiento y Discapacidad (OMS, 2001) y concebida desde un modelo ecológico y biopsicosocial de la discapacidad, lo cual favorece en gran medida la planificación de procedimientos de intervención y servicios (Badía-Corbella, 2007):

"La Parálisis Cerebral describe un grupo de trastornos permanentes del desarrollo del movimiento y de la postura, que causan limitaciones en la actividad y que son atribuidos a alteraciones no progresivas ocurridas en el desarrollo cerebral del feto o del lactante. Los trastornos motores de la parálisis cerebral están a menudo acompañados por alteraciones de la sensación, percepción, cognición, comunicación y conducta, por epilepsia y por problemas musculoesqueléticos secundarios" (Rosenbaum et al., 2007: 9).

Las personas con PC envejecen, y entender los síntomas y complicaciones subyacentes a su discapacidad y cómo influyen en su expectativa de vida resulta crucial (Coppus, 2013). Este colectivo se ve afectado por dificultades de salud ligadas a la edad, a las que se suma la morbilidad y circunstancias secundarias asociadas a su discapacidad, sirva de ejemplo la gran pérdida de movilidad (Morgan y McGinley, 2014). A medida que se envejece se sufre un incremento de la morbilidad y de la dependencia, que afecta a la calidad de vida de estas personas (Badía-Corbella et al., 2013); dada esta nueva realidad demográfica, es preciso conocer el estado de publicaciones en esta área para evaluar si desde la investigación se puede dar respuesta a los retos a los que se enfrentan las personas con PC, sus familiares y los profesionales que les atienden.

Por tanto, el objetivo general de esta revisión es analizar de manera sistemática las publicaciones en materia de envejecimiento de las personas con parálisis cerebral. Se pretende que esta revisión suponga "un trabajo reflexivo y comprensivo, en el cual se lleva a cabo un análisis histórico-crítico interdisciplinar de un tema" (Fernández-Ríos y Buela-Casal, 2009: 331) y que sirva como punto de partida para plantear la necesidad de nuevos estudios en la materia. Los objetivos concretos que se desprenden del general y que son los que guiarán esta revisión son los que siguen:

1. Efectuar una aproximación al volumen y tipo de publicaciones en materia de envejecimiento en personas con parálisis cerebral.

2. Examinar el tipo de metodología utilizada en las investigaciones encontradas.

3. Identificar y clasificar las áreas temáticas de estudio en las que se centran las publicaciones dentro de esta materia con el fin de conocer qué suscita el interés de los investigadores.

4. Comprobar la edad en la que los diferentes estudios sitúan el comienzo del proceso de envejecimiento en personas con PC. 


\section{Método}

En este segundo apartado se detalla el método llevado a cabo para realizar la revisión, con el fin de que esta sea replicable, para ello se detallan las fuentes y los descriptores utilizados, los criterios de inclusión y exclusión de los documentos hallados y las ubicaciones de estos en las distintas revistas.

\subsection{Protocolo y registro}

Las revisiones sistemáticas son un recurso metodológico y una herramienta de investigación que nos posibilita información y actualización temática sin necesidad de invertir tiempo y recursos (Perestelo-Pérez, 2013). La presente revisión se ha centrado en exclusividad en los trabajos que han sido publicados como artículos en revistas, localizados en las bases de datos ERIC, DIALNET, Medline y PsycINFO, consideradas las más prolíferas en la agrupación de publicaciones en ciencias sociales y de la salud. Para evaluar los trabajos se ha utilizado el procedimiento propio de las revisiones del sistema PRISMA. La búsqueda realizada para la revisión abarca el periodo de los dieciocho últimos años, lo que permite valorar la progresión de investigación en el campo de interés. Los criterios de inclusión han sido, por tanto, artículos de revista publicados entre enero del año 2000 y enero de 2018 sobre PC en personas mayores de edad (18 años). Como criterio de exclusión se omiten los artículos que abordan otra discapacidad en la etapa adulta o la PC en la etapa infantil o adolescente. En la exploración se cruzaron como términos críticos las palabras clave: "cerebral palsy" por un lado, y tanto "aging" como "ageing" unidas por AND con el fin de obtener publicaciones que abordasen el envejecimiento tanto americanas como británicas; y sus correspondientes términos en castellano: "parálisis cerebral" y "envejecimiento".

\subsection{Fuentes de información y estrategias de búsqueda}

Tal y como puede verse en la figura 1, de las 161 referencias halladas, se han seleccionado las que hacían referencia directa al envejecimiento de las personas con PC. Se han excluido las publicaciones que no abordaban en tema del envejecimiento en este colectivo, es decir, artículos que tratan otras etapas vitales de la PC o que abordan el envejecimiento en discapacidad en general. Además, se han eliminado aquellas que aparecían duplicadas en las bases de datos.

De este modo se han recuperado 61 referencias, de las cuales 36 aparecen en la base de datos Medline. Este dato indica el posible interés de las condiciones médicas en este tipo de discapacidad que se aborda en el análisis posterior, 18 referencias aparecen en PsycINFO, 6 en ERIC y 2 en Dialnet.

Se han eliminado 14 referencias por no aparecer completas o por abordar la PC en población infantil $(n=3)$, por lo que finalmente se han recuperado un total de 47 artículos completos que cumplían con todos los criterios establecidos. 
Figura 1: Flujo de selección de artículos

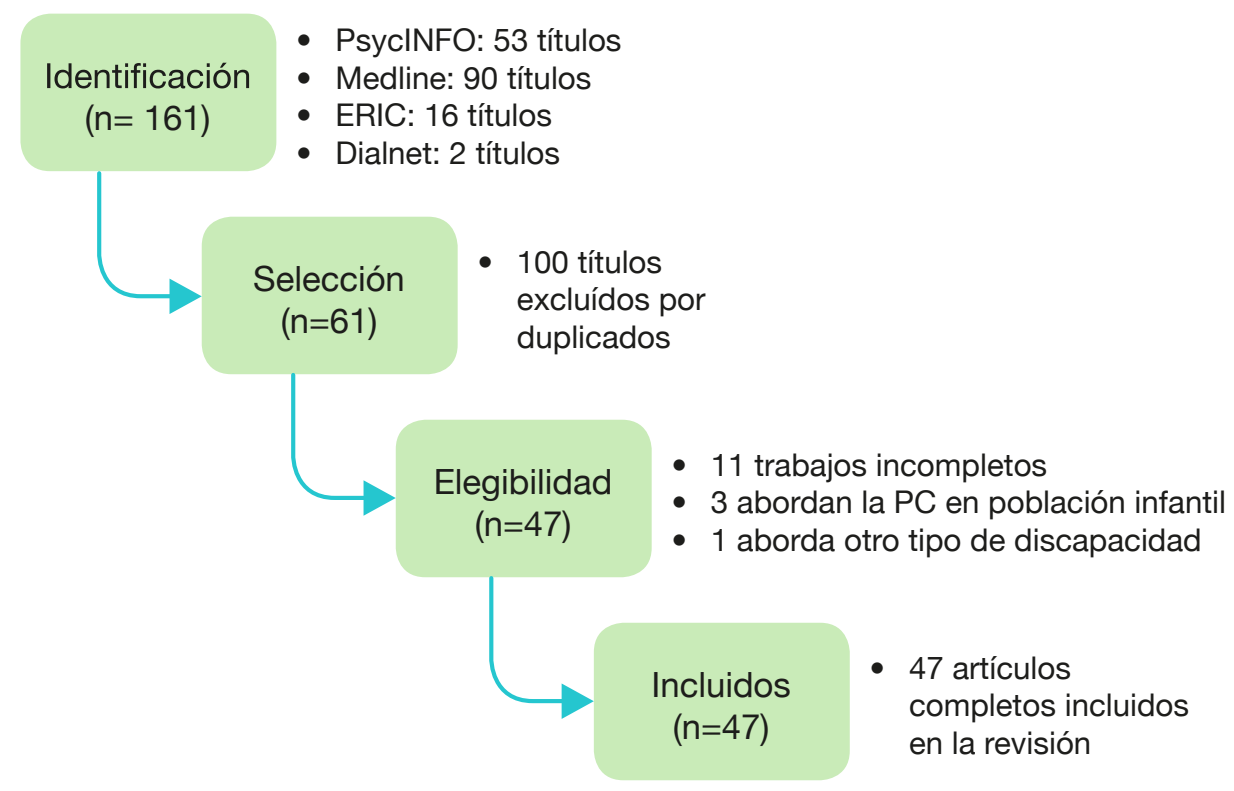

Fuente: elaboración propia (basada en Moher et al., 2009).

\subsection{Evaluación de la calidad, y análisis de datos y síntesis}

En esta búsqueda se destaca la falta de informes oficiales y/o estadísticas. En cuanto a los artículos en publicaciones periódicas, en las referencias aparecen 24 publicaciones diferentes. La revista que más artículos aglutina es Developmental Medicine And Child Neurology con un total de 13 referencias sobre el tema entre los años 2001 y 2016, en segundo lugar, Disability and Rehabilitation: An International, Multidisciplinary Journal con un total de 7 referencias entre los años 2003 y 2013 y, en tercer lugar, aparece Journal of Intellectual \& Developmental Disability con 5 referencias entre 2007 y 2014.

Dichas publicaciones están especializadas en áreas como: 1) la discapacidad y el entorno rehabilitador; 2) aspectos médicos, centrados principalmente en el área neurológica 3) disciplinas psicológicas y 4) envejecimiento.

En lo referente al examen del tipo y volumen de publicaciones se observa que priman las publicaciones de carácter médico y/o rehabilitador y que los años en los que se han publicado más investigaciones han sido los años 2007 y 2009, sin embargo, no hay una tendencia temporal clara que marque un auge de publicaciones en esta área. 


\section{Resultados}

El tercer apartado aborda los resultados encontrados en la revisión realizada. Se estructuran y organizan las publicaciones con base a dos criterios, por un lado, la metodología utilizada por parte de los investigadores, ya que este es uno de los objetivos planteados en el presente trabajo. En concreto se plantean dos tipos de publicaciones, las teóricas y las empíricas. El segundo criterio de organización son las áreas de interés que se estudian.

\subsection{Metodología}

Tal y como queda reflejado en la tabla 1, las publicaciones teóricas suponen un $44,60 \%$ del total (en concreto 21 de las 47 publicaciones revisadas), estas nos ofrecen una visión actual y estado de la cuestión sobre el tema. De estas 21 publicaciones sólo 3 son informes, siendo las 18 restantes $(38,29 \%)$ revisiones teóricas, de las cuales solo 6 son revisiones sistemáticas y otras 12 realizan búsquedas que reflejan un estado de la cuestión, pero sin un método sistemático y replicable en su procedimiento.

Seguidamente, las 26 publicaciones restantes son empíricas, es decir, en ellas se presenta muestra de personas con PC para ofrecer algún tipo de datos (55,31\% del total de la revisión). Dentro de esta metodología pueden clasificarse atendiendo a las siguientes categorías: 12 son estudios con metodología cuantitativa (el $31,91 \%), 11$ presentan naturaleza cualitativa (27,65\%) y 2 estudios combinan ambas metodologías $(4,25 \%)$.

\begin{tabular}{l}
\hline Tabla 1. Distribución de las publicaciones \\
\begin{tabular}{|l|c|c|}
\hline Metodología & $\mathbf{N}^{\circ}$ & $\%$ \\
\hline Teórica: & 21 & 44,60 \\
\hline - Revisiones & 18 & 38,29 \\
\hline - Informes & 3 & 6,38 \\
\hline Empírica & 26 & 55,31 \\
\hline - Metodología cualitativa & 11 & 23,40 \\
\hline - Metodología cuantitativa & 13 & 27,65 \\
\hline - Metodología mixta & 2 & 4,25 \\
\hline Total & $\mathbf{4 7}$ & $\mathbf{1 0 0}$ \\
\hline
\end{tabular}
\end{tabular}

Fuente: elaboración propia.

\subsubsection{Estudios teóricos}

Los estudios que abordan la temática desde una revisión de la literatura o estado de la cuestión se plasman en la tabla 2, donde se destaca el objetivo que persigue cada una de las publicaciones. 


\begin{tabular}{|c|c|}
\hline Autor/Año & Objetivo \\
\hline Brunton y Rice (2012) & Realizar una revisión crítica de la literatura relacionada con la fatiga en PC. \\
\hline Cans et al. (2000) & $\begin{array}{l}\text { Monitorizar las tasas de prevalencia, especialmente dentro de los subgrupos (peso al nacer, } \\
\text { tipo clínico). }\end{array}$ \\
\hline Frisch y Msall (2013) & $\begin{array}{l}\text { Revisar la literatura que evalúa los resultados en adultos con PC en el marco del modelo de } \\
\text { habilitación de la Clasificación Internacional del Funcionamiento (CIF), Discapacidad y Salud. }\end{array}$ \\
\hline $\begin{array}{l}\text { González-Alonso et al. } \\
\text { (2017) }\end{array}$ & Recopilar información sobre PC y envejecimiento: necesidades percibidas y calidad de vida. \\
\hline Haak et al. (2009) & $\begin{array}{l}\text { Resumir lo que se conoce de la epidemiología de la PC a lo largo de la vida, comenzando con } \\
\text { la mortalidad y la esperanza de vida y qué se sabe sobre el funcionamiento, la capacidad y la } \\
\text { calidad de vida de los adultos con PC. }\end{array}$ \\
\hline Haddad et al. (2007) & $\begin{array}{l}\text { Revisar el desarrollo de comorbilidades en pacientes con PC y explorar áreas potenciales para } \\
\text { la intervención de tratamientos farmacéuticos. }\end{array}$ \\
\hline Hemsley et al. (2007b) & $\begin{array}{l}\text { Resumir la literatura relacionada con cuidadores remunerados de adultos con PC y } \\
\text { necesidades complejas. }\end{array}$ \\
\hline Horstmann et al. (2009) & $\begin{array}{l}\text { Resumir la literatura que aborda la atención de salud para el adulto con problemas ortopédicos } \\
\text { relacionados con la PC. }\end{array}$ \\
\hline Klingbeil et al. (2004) & $\begin{array}{l}\text { Destacar problemas importantes en el cuidado de personas que están envejeciendo y tienen } \\
\text { una discapacidad. }\end{array}$ \\
\hline McPhee et al. (2016) & $\begin{array}{l}\text { Describir la experiencia de fatiga en adultos con PC y determinar si el nivel de actividad física, } \\
\text { el tiempo sedentario, la edad o la composición corporal pueden predecir la fatiga en adultos } \\
\text { con PC. }\end{array}$ \\
\hline Morgan y McGinley (2014) & $\begin{array}{l}\text { Identificar, evaluar y sintetizar la evidencia que describe la disminución de la marcha en adultos } \\
\text { con PC. }\end{array}$ \\
\hline Peterson et al. (2013) & $\begin{array}{l}\text { Discutir las características fenotípicas que coinciden con el envejecimiento, la obesidad y los } \\
\text { trastornos cardiometabólicos. }\end{array}$ \\
\hline Peterson et al. (2012) & $\begin{array}{l}\text { Discutir los mecanismos y las consecuencias secundarias asociadas con el comportamiento } \\
\text { sedentario crónico, la obesidad, el envejecimiento y la espasticidad muscular. }\end{array}$ \\
\hline Platt (2016) & Recalcar la importancia de establecer registros longitudinales específicos de PC. \\
\hline $\begin{array}{l}\text { Pueyo-Benito y Vendrell- } \\
\text { Gómez, (2002) }\end{array}$ & $\begin{array}{l}\text { Revisar los trabajos neuropsicológicos que han examinado el rendimiento cognitivo general y el } \\
\text { rendimiento específico (lenguaje, memoria, atención y funciones visoespaciales). }\end{array}$ \\
\hline Shortland (2009) & Determinar el volumen de masa muscular en personas con PC adultas y su relevancia. \\
\hline Svien et al. (2008) & $\begin{array}{l}\text { Presentar la literatura actual sobre los efectos que el envejecimiento tiene en los adultos con } \\
\text { PC. }\end{array}$ \\
\hline Thorpe (2009) & $\begin{array}{l}\text { Abordar el estado de la ciencia con respecto a la actividad física y la condición física para } \\
\text { adultos con PC y cómo el entrenamiento físico se relaciona con la actividad física y la salud en } \\
\text { esta población. }\end{array}$ \\
\hline Tosi et al. (2009) & $\begin{array}{l}\text { Resumir los hallazgos de un taller multidisciplinario, patrocinado por la Fundación Internacional } \\
\text { para la Investigación de la PC, la Academia Americana para la PC y la Medicina del Desarrollo. }\end{array}$ \\
\hline Turk (2009) & Recopilar información sobre el envejecimiento en PC. \\
\hline Turk et al. (2001) & Desarrollar una mejor apreciación del efecto que ha tenido la PC en la vida de las mujeres. \\
\hline
\end{tabular}

Fuente: elaboración propia. 
En primer lugar, cabe apuntar que varias de las revisiones abordadas enfatizan la necesidad de estudios epidemiológicos detallados y rigurosos de manera que permitan ver la evolución de la PC a lo largo del ciclo vital de los sujetos. Aquí se pone de relieve esta carencia de estudios epidemiológicos ya que en esta revisión sólo se han encontrado dos estudios de estas características (Haak et al., 2009; Cans et al., 2000).

En segundo lugar, destaca la homogeneidad en la detección de necesidades de investigación expuestas por Turk (2009) y coincidentes con otros autores:

- Dada la ausencia de información longitudinal no está claro si la relación entre la gravedad y la edad es continua.

- El estado de la ciencia en relación con la salud de los adultos con PC ha mejorado en los últimos años, aunque aún se dispone de menos información acerca de los adultos que de los niños (González-Alonso et al., 2017).

- La mayoría de los estudios continúan informando sobre un número muy limitado de participantes.

- Se necesita información adicional sobre el manejo de dolor crónico, el impacto de las intervenciones comunes durante toda la vida, los cambios relacionados con la edad, la influencia de la gravedad de la enfermedad y el deterioro cognitivo.

- Se precisa una mayor comprensión de los problemas de salud mental asociados con, o derivados de la discapacidad (Platt, 2016).

- Hay una falta de información sobre los costes y sobre los cambios de rendimiento en el tiempo y su relación e influencia con las remodelaciones de vivienda, empleo / jubilación, necesidades de apoyo, asistencia sanitaria y seguro de asistencia.

- Tampoco hay información suficiente sobre la eficacia del cribado y las actividades de promoción de la salud, especialmente el ejercicio, durante toda la vida.

- Se precisa una metodología de investigación cualitativa, que explore los aspectos sociales y psicológicos (Hemsley et al., 2007a).

A pesar de la relevancia de estas conclusiones, cabe apuntar que estas necesidades de investigación señaladas en las revisiones halladas son extensibles a otras discapacidades y no son exclusivas del estudio del envejecimiento en PC (Del Barrio et al., 2016).

\subsubsection{Estudios empíricos}

Por lo que respecta a los estudios empíricos, en la tabla 3 se muestra una clasificación de aquellos estudios que presentan muestras de personas con PC, el número de sujetos que incluye cada publicación y el objetivo que plantea. 


\begin{tabular}{|c|c|c|}
\hline Autor/Año & $\mathbf{N}$ & Objetivo \\
\hline $\begin{array}{l}\text { Andersson y Mattsson } \\
(2001)\end{array}$ & 221 & $\begin{array}{l}\text { Describir los problemas y los recursos de los adultos con PC con especial énfasis en la } \\
\text { locomoción. }\end{array}$ \\
\hline Badía-Corbella et al. (2013) & 6 & $\begin{array}{l}\text { Determinar la calidad de vida en adultos con PC durante el proceso de envejecimiento y analizar } \\
\text { el efecto de la función motora. }\end{array}$ \\
\hline Balandin et al. (2006) & 20 & $\begin{array}{l}\text { Comparar los niveles de soledad entre dos grupos de adultos con PC en función del tipo de } \\
\text { comunicación. }\end{array}$ \\
\hline Balandin et al. (2009) & 32 & $\begin{array}{l}\text { Identificar cambios en la capacidad de deglución de los adultos PC que pueden afectar su } \\
\text { salud, seguridad y bienestar. }\end{array}$ \\
\hline Ballin y Balandin (2007) & 7 & Determinar niveles de soledad en adultos con PC. \\
\hline Benner et al. (2017) & 49 & $\begin{array}{l}\text { Describir el cambio longitudinal en la salud percibida, la presencia de problemas de salud y el } \\
\text { nivel funcional en adultos con PC. }\end{array}$ \\
\hline Bottos et al. (2001) & 72 & Examinar la evolución de los individuos con PC desde la infancia hasta la edad adulta. \\
\hline Chadwick y Jolliffe (2009) & 101 & Investigar la disfagia en personas con discapacidad incluyendo la PC. \\
\hline Dark et al. (2016) & 22 & $\begin{array}{l}\text { Comprender mejor cómo los adultos con PC experimentan cambios en sus capacidades de } \\
\text { comunicación a medida que envejecen y el impacto psicosocial posterior. }\end{array}$ \\
\hline Galambos et al. (2007) & 76 & Examinar la edad subjetiva (qué edad siente) y las variables asociadas al envejecimiento. \\
\hline $\begin{array}{l}\text { Goodwin y Compton } \\
\text { (2004) }\end{array}$ & 2 & Comprender las experiencias de la actividad física y el envejecimiento con PC. \\
\hline Heller et al. (2002) & 83 & $\begin{array}{l}\text { Examinar el impacto de los factores ambientales y las actitudes de los cuidadores sobre la } \\
\text { participación en el ejercicio en adultos con PC utilizando un modelo socio-cognitivo. }\end{array}$ \\
\hline Hemsley et al. (2007a) & 6 & $\begin{array}{l}\text { Explorar las opiniones de los cuidadores familiares de adultos con PC sobre sus funciones en el } \\
\text { entorno hospitalario y lo que les ayudaría en estas funciones. }\end{array}$ \\
\hline Horsman et al. (2010) & 12 & Entender las experiencias únicas y vividas de adultos que envejecen con PC. \\
\hline Jones (2009) & 1 & $\begin{array}{l}\text { Describir la experiencia de vida de una mujer de } 64 \text { años que está envejeciendo con PC y otras } \\
\text { discapacidades múltiples. }\end{array}$ \\
\hline Lifshitz et al. (2008) & 32 & $\begin{array}{l}\text { Estudiar las diferencias en el fenómeno del envejecimiento entre adultos con discapacidad } \\
\text { (grupo de PC) que viven en residencias comunitarias frente a sus pares en centros de atención } \\
\text { residencial. }\end{array}$ \\
\hline Low Choy et al. (2003) & 22 & $\begin{array}{l}\text { Investigar la eficacia de un programa de intervención en estaciones de trabajo para mejorar la } \\
\text { capacidad funcional y la flexibilidad en pacientes de edad avanzada con PC. }\end{array}$ \\
\hline Michelsen et al. (2006) & 416 & Examinar la integración social de personas con PC. \\
\hline Mitchell et al. (2006) & 262 & Investigar el papel del envejecimiento y la discapacidad en el estado laboral a lo largo de la vida. \\
\hline Moll y Cott (2012) & 9 & $\begin{array}{l}\text { Examinar la experiencia de la normalización a través de la rehabilitación para personas que } \\
\text { crecen y envejecen con PC. }\end{array}$ \\
\hline Morgan et al. (2014) & 6 & $\begin{array}{l}\text { Explorar en profundidad la experiencia y el impacto del acceso a los servicios de salud para } \\
\text { abordar el cambio de movilidad en adultos con PC. }\end{array}$ \\
\hline Morgan et al. (2015) & 34 & $\begin{array}{l}\text { Describir las causas percibidas, las influencias ambientales y las consecuencias de las caídas } \\
\text { en adultos ambulantes con PC. }\end{array}$ \\
\hline Mudge et al. (2016) & 37 & $\begin{array}{l}\text { Mejorar la comprensión de las experiencias de envejecimiento con PC en la edad adulta, con un } \\
\text { enfoque particular en las experiencias con servicios de salud. }\end{array}$ \\
\hline Sandström et al. (2009) & 22 & $\begin{array}{l}\text { Obtener una comprensión más profunda de cómo los adultos con PC experimentan la } \\
\text { fisioterapia y la actividad física en una perspectiva desde la infancia hasta la edad adulta. }\end{array}$ \\
\hline $\begin{array}{l}\text { Tarsuslu y Livanelioglu } \\
(2010)\end{array}$ & 45 & $\begin{array}{l}\text { Investigar la relación entre la calidad de vida relacionada con la salud y el estado funcional en } \\
\text { adultos jóvenes o adultos con PC. }\end{array}$ \\
\hline Taylor et al. (2004) & 10 & $\begin{array}{l}\text { Indicar si un programa de entrenamiento de fuerza de resistencia progresiva basado en la } \\
\text { comunidad podría mejorar la fuerza muscular y la actividad funcional en un grupo de adultos } \\
\text { con PC con altas necesidades de apoyo. }\end{array}$ \\
\hline
\end{tabular}

Fuente: elaboración propia. 
Dentro de los estudios empíricos, se distingue entre los estudios cualitativos, los estudios cuantitativos y aquellos que realizan una combinación de ambos.

Se incluyen en la categoría de estudios empíricos cualitativos aquellos que emplean estudios de caso, encuestas y entrevistas en profundidad a las personas con PC o sus familiares y cuidadores.

En esta revisión solo se ha encontrado un estudio de caso único (Jones, 2009), aunque el estudio de Goodwin y Compton (2004) examina a través de entrevistas en profundidad semiestructuradas y sus respectivas trascripciones las percepciones de 7 mujeres acerca de sus experiencias en actividades físicas y en el proceso de envejecer con una discapacidad.

Así mismo encontramos otros estudios que recurren a la entrevista en profundidad semiestructurada y elaborada ad hoc según los objetivos de la investigación (Sandström et al., 2009; Horsman et al., 2010). Esta metodología permite obtener una visión más amplia de las necesidades expuestas por las personas y el modo en que estas son vividas.

Retomando las temáticas principales encontradas en la revisión, los estudios cualitativos abordan aspectos psicosociales como la soledad (Ballin y Balandin, 2007) y aspectos médicos y rehabilitadores como la capacidad de deglución (Balandin et al., 2009) el estado funcional y la actividad física (Bottos et al., 2001; Sandström et al. 2009). Cabe destacar que la información se extrae en algunos estudios de terceras personas (familiares o cuidadores) que aportan información acerca de las personas con PC mediante entrevistas o encuestas y no directamente de las personas con PC (Cleaver et al. 2008).

El segundo tipo dentro de los estudios empíricos son los cuantitativos, aquellos que investigan el envejecimiento de personas con PC mediante el uso de instrumentos de evaluación estandarizados.

En estos estudios es frecuente encontrar unas cuestiones metodológicas sujetas a mejora que deberían solventarse en estudios posteriores para lograr así una mayor rigurosidad y verificabilidad en el estudio del proceso de envejecimiento de personas con PC. Esta situación es extensible al estudio del proceso de envejecimiento en otro tipo de discapacidad (Alcedo et al. 2017).

En primer lugar, se detectan problemas en cuanto a la muestra utilizada en estos estudios. Por un lado, se hallan estudios con muestras reducidas en los que se indica que se debería ampliar la muestra (Ballin et al., 2007). Además, también es frecuente que exista una dificultad para lograr grupos de control comparables, esto se debe en parte a que la muestra no se selecciona aleatoriamente, sino que se trata de una muestra de conveniencia y dada la alta institucionalización de las personas con PC muchas veces se acude solo a las instituciones para acceder a la muestra (Balandin et al., 2009; Badía-Corbella et al., 2013). Esto dificulta la posterior generalización de resultados.

Por otra parte, como ya se ha comentado con anterioridad en referencia a los estudios teóricos, este campo de investigación adolece de estudios longitudinales que permitan ver con claridad las cuestiones relacionadas con el propio proceso de envejecimiento, hecho fundamental en un proceso vital del que se requiere conocer la evolución de los casos y extraer comparaciones intrasujeto. 
En lo referente a los instrumentos de evaluación, en el caso del estudio de la PC, como ya se ha visto, el peso del modelo médico es notable. Por ello, encontramos que es frecuente el uso del Gross Motor Function Classification System for CP (GMFCS) para clasificar a los sujetos y ver cómo cambian según tramos de edad (Morgan et al., 2014; Tarsuslu y Livanelioglu, 2010; Taylor et al., 2004; Badía-Corbella, et al. 2013). En consonancia con lo anterior, la Physical Mobility Scale (PMS), es un instrumento muy utilizado (Low Choy et al., 2003; Tarsuslu y Livanelioglu, 2010) o la Functional Independence Measure (FIM) (Tarsuslu y Livanelioglu, 2010). En el caso de los estudios sobre la soledad de los ancianos con PC se ha creado y desarrollado la UCLA Loneliness Scale (Balandin et al., 2006).

En cuanto a los estudios mixtos, aquellos que combinan metodología cuantitativa y cualitativa, se encuentran las publicaciones que acompañan las entrevistas en profundidad con las pruebas 36-Item Short Form Health Survey y el Barthel Index (Benner et al., 2017), o con la ya comentada GMFCS (Morgan et al., 2015).

\section{2. Áreas temáticas de interés}

Siguiendo los objetivos planteados en la presente revisión, se analizan las áreas temáticas que han suscitado el interés de los investigadores. En términos generales, tal y como se detalla en la tabla 4, las publicaciones revisadas se clasifican en dos áreas temáticas, aspectos psicosociales y aspectos médicos y rehabilitadores; siendo esta última en la que se concentran el mayor número de artículos.

\begin{tabular}{l}
\hline Tabla 4. Distribución de publicaciones por temática principal \\
\begin{tabular}{|l|c|c|}
\hline Temática principal & No & $\%$ \\
\hline Aspectos psicosociales & 9 & 19,14 \\
\hline - Soledad y comunicación & 3 & 6,38 \\
\hline - Experiencias hospitalarias y cuidadores & 3 & 6,38 \\
\hline - Integración social y empleabilidad & 1 & 2,12 \\
\hline - Necesidades y calidad de vida & 2 & 4,25 \\
\hline Aspectos médicos y rehabilitadores & 38 & 80,85 \\
\hline - Envejecimiento general & 13 & 27,65 \\
\hline - Salud y necesidades médicas & 11 & 23,40 \\
\hline - Estado funcional y actividad física & 14 & 29,78 \\
\hline
\end{tabular}
\end{tabular}

Fuente: elaboración propia.

El primer grupo descrito en este estudio es el menos numeroso, este aborda el proceso de envejecimiento desde una perspectiva más psicosocial no centrándose tanto en las variables asociadas al envejecimiento sino en el modo en el que la persona experimenta. Entre los temas más frecuentes se encuentra la soledad asociada a esta etapa de la vida y el uso de la comunicación alternativa (Balandin et al., 2006; Ballin y Balandin, 2007) ya que según estos autores en el proceso de envejecimiento las personas con PC experimentan más soledad que el mismo grupo de edad sin discapacidad, derivada esta de sus problemas de comunicación y de sus limitaciones funcionales. 
En este primer grupo se encuentran también estudios que abordan las experiencias derivadas de las hospitalizaciones de larga duración y cómo son estas vividas por los cuidadores principales y/o personas de apoyo (Hemsley et al., 2007a; Hemsley et al., 2007b) recalcando la necesidad de generar estrategias de apoyo a los cuidadores mayores de los adultos con discapacidad que no pueden hablar en el hospital, y concebir otras alternativas para la prestación de apoyo a estos adultos durante los períodos de hospitalización.

Por último, dentro de este grupo de aspectos psicosociales se sitúan los artículos que abordan la integración social de personas con PC (Michelsen et al., 2006a), las tasas de empleo de este colectivo (Mitchell et al., 2006) y los aspectos psicosociales propiamente dichos del envejecimiento, tales como necesidades y calidad de vida (Horsman et al., 2010; Badía-Corbella et al., 2013). Concluyendo por un lado que no se han encontrado signos de una mayor integración social en las últimas dos o tres décadas en Dinamarca, que la educación universitaria mejora las tasas de empleo para las personas con discapacidad en sus 20 y 30 años, pero no evita la pérdida significativa de empleo a partir de los 40 años. Y, por último, que los adultos con PC necesitan un mayor conocimiento y entendimiento para mejorar la toma de decisiones sobre su salud y que en este proceso de envejecimiento hay una pérdida de calidad de vida, sobre todo en autodeterminación y derechos.

Dentro del segundo grupo de publicaciones, que representan el $80,25 \%$ de la revisión, centradas en los aspectos médicos y/o rehabilitadores, se encuentran publicaciones que abordan el envejecimiento de una forma general. Varias publicaciones coinciden en que el proceso de envejecimiento, inevitablemente, debe interactuar con el trastorno motor (Haak et al., 2009; Klingbeil et al., 2004), así mismo, se destaca la importancia de explorar las discapacidades motoras como fuente de diversidad en la edad subjetiva de los jóvenes durante la transición a la edad adulta (Galambos et al., 2007).

En línea con lo anterior, se hallan publicaciones que abordan aspectos de la salud y las necesidades médicas. Así, se exploran los cambios neuromusculares y neuromuscoesqueléticos asociados a la edad (Tosi et al., 2009) tales como dolor, fatiga crónica, y una inmovilidad prematura y decaimiento de la función. Además, se explora la disfagia encontrándose una prevalencia actual en ancianos con PC del 8,15\% (Chadwick y Jolliffe, 2009); la farmacología de este colectivo, que indica que las condiciones médicas crónicas que generalmente se observan en la población general adulta mayor pueden aparecer tempranamente en los pacientes con PC (Haddad et al.,2007), y la ortopedia, encontrándose que la mayoría de los problemas encontrados en adultos mayores están asociadas a los residuos de las cuestiones de la infancia, en particular deformidades asociadas a las contracturas (Horstmann et al., 2009). Dentro de este grupo también hay publicaciones que listan las necesidades médicas y aspectos generales de salud de este colectivo (Turk, 2009) y de las mujeres en concreto (Turk et al. 2001).

Por último, siendo el grupo de publicaciones más numeroso y por tanto el área temática que más interés despierta en los investigadores, se encuentran publicaciones que abordan el estado funcional y la actividad física en personas mayores con PC, coincidiendo en afirmar que el declive comienza a mediados de la veintena y presenta una aceleración del deterioro después de la séptima década. Es posible que el déficit muscular característico de los jóvenes con PC, junto con el descenso de las propiedades del músculo en la edad adulta, contribuyan a una pérdida temprana de la movilidad en este grupo (Shortland, 2009; Tarsuslu y Livanelioglu, 2010). 


\section{Puntos de corte por edad}

En este apartado se aborda el último objetivo planteado en la revisión que se centra en identificar en qué edad se sitúa el consenso sobre el inicio del proceso de envejecimiento en personas con PC.

A continuación, en la tabla 5, se presentan algunos de los estudios más relevantes y sus puntos de corte por edad. El límite inferior más repetido son 40 años, sin embargo, este umbral es variable, por lo que se comprueba que no existe un consenso bien definido sobre a qué edad comienza el proceso de envejecimiento en este colectivo, aunque lo que sí parece claro es que este es prematuro en personas con PC (Klingbeil et al., 2004).

Tabla 5. Puntos de corte por edad, umbral inferior

\begin{tabular}{|l|l|}
\hline Estudio & Inferior \\
\hline Badía-Corbella et al. (2013) & 40 \\
\hline Balandin et al. (2006) & 40 \\
\hline Balandin et al. (2009) & 30 \\
\hline Ballin y Balandin (2007) & 40 \\
\hline Benner et al., (2017) & 49 \\
\hline Cleaver et al., (2008) & 45 \\
\hline Dark et al, (2016) & 40 \\
\hline Lifshitz et al. (2008) & 40 \\
\hline Low Choy et al. (2003) & 37 \\
\hline Morgan et al., (2014) & 35 \\
\hline Mudge et al., (2016) & 37 \\
\hline Sandström et al. (2009) & 35 \\
\hline
\end{tabular}

Fuente: elaboración propia.

\section{Discusión y conclusiones}

Tras el análisis de las publicaciones en el apartado de resultados, cierra la revisión la discusión de los resultados extraídos del análisis y la conclusión derivada de estos.

Tal y como se plantea en la introducción, las publicaciones indican que el aumento en la esperanza de vida de las personas con PC ha seguido una tendencia paralela a la encontrada en la población general (Coppus, 2013). Por ello los adultos con PC que envejecen necesitan acceso a servicios de salud para satisfacer sus 
necesidades cambiantes de esta etapa vital (Morgan et al., 2014). Esta población experimenta cambios múltiples y funcionales a medida que envejecen (Dark et al., 2016), que influyen en la salud percibida y en una disminución del nivel funcional (Benner et al., 2017).

Las publicaciones halladas en la revisión describen un proceso de envejecimiento en el que destacan las deficiencias orgánicas relacionadas con la PC, así como la gravedad del impacto incapacitante que aumenta con la edad (Mudge et al., 2016). Esta etapa vital en las personas con PC está asociada a comportamientos sedentarios extremos que acarrean una progresión acelerada característica de la patología muscular (Peterson et al., 2012) que a su vez conlleva un declive en la capacidad funcional (Taylor et al., 2004). Esta disminución prematura de la función también se atribuye a debilidad, espasticidad y anomalías ortopédicas, así como a dolor crónico y fatiga (Brunton y Rice, 2012; Peterson et al., 2013; Benner et al., 2017). En este sentido, a medida que envejecen, pierden muchos de los logros que adquirieron en la rehabilitación (Moll y Cott, 2013). Asimismo, Peterson et al. (2013) y McPhee et al., (2016) coinciden en que se ha llevado a cabo muy poca investigación acerca de la obesidad como mediadora de la comorbilidad secundaria en esta población. Otra condición común en la PC y que precisa más investigación es la disfagia (Chadwick y Jolliffe, 2009). Igualmente deben estudiarse los cambios que afectan al modo de comunicarse y los métodos que permiten el desarrollo y el mantenimiento de las relaciones, la participación comunicativa y la calidad de vida (Dark et al., 2016).

Además del análisis sintético sobre el contenido de las publicaciones en la caracterización del proceso de envejecimiento extraído de las publicaciones, el presente trabajo se plantea objetivos propios de la revisión realizada que aporten una visión general de la investigación en el área y permitan conocer a nuevos trabajos en esta temática qué se ha publicado hasta el momento y qué necesidades de investigación hay en el área.

En primer lugar, por lo que respecta al tipo de publicaciones, se observa una mayoría de publicaciones de carácter médico publicadas en revistas de esta temática. Respecto al volumen, no se encuentra una tendencia de progresión o decrecimiento de resultados en los últimos 18 años, a pesar de que la revolución demográfica crea una demanda de necesidad de respuestas del colectivo. Se evidencia una escasez de estudios en castellano sobre el tema ( 2 de 47 , un $4,27 \%$ ), falta de informes oficiales y/o estadísticas y la única presencia de dos manuales sobre envejecimiento en PC.

En segundo lugar, en cuanto a la metodología de los estudios, en esta área los estudios teóricos tienen menor presencia que los que ofrecen datos. Dentro de los primeros, cabe resaltar la mayoría de revisiones no sistemáticas frente a la escasez de informes. Los estudios que ofrecen datos presentan porcentajes similares en metodología cualitativa y cuantitativa, pero con la existencia de solo dos mixtos. Los problemas metodológicos encontrados son comunes a otras áreas de investigación en discapacidad, entre los que destaca la falta de estudios longitudinales, y problemas con el tamaño y la configuración de la muestra.

En tercer lugar, se observa la existencia de dos líneas de investigación paralelas, por un lado, una primera que estudia aspectos psicosociales (soledad y comunicación, experiencias hospitalarias y cuidadores e integración social, empleabilidad y calidad de vida) y una segunda línea más fructífera en estudios que la anterior que se centra en aspectos médicos y rehabilitadores (envejecimiento general, salud y necesidades médicas y estado funcional y actividad física). Dentro de este grupo predominante de estudios, el mayor porcentaje de las publicaciones versa sobre el estado funcional y la actividad física de personas con PC. 
Por último, en lo referente a la edad en la que se cifra el inicio del proceso de envejecimiento el único consenso es que este es prematuro en el colectivo de referencia y se sitúa entre los 30 y los 45 años, siendo el punto de corte inferior más frecuente en las publicaciones los 40 años.

En consonancia con todo lo anterior, se concluye que el área de publicaciones en temática de envejecimiento en personas con discapacidad es heterogénea y en ella prima la perspectiva médica rehabilitadora, dentro de la cual se analizan las deficiencias y patologías del proceso de envejecimiento de este colectivo y las peculiaridades de este con respecto a este proceso en la población normativa. Además, derivado de lo anterior, se concluye que hay menores publicaciones que ahonden en el cómo la persona percibe este proceso, su calidad de vida y la repercusión de las citadas deficiencias y patologías en la vida de la persona, siendo esta línea necesaria para poder atender con calidad y de manera interdisciplinar a las demandas que se generan en esta etapa.

El área presenta debilidades metodológicas que deben ser mejoradas, sino resueltas, en las investigaciones que se realicen a futuro en la temática, con estudios longitudinales que aporten evidencias sobre la relación entre edad y gravedad, muestras más amplias, y mayor consenso entre los investigadores a los criterios de inclusión y exclusión de los sujetos en las muestras con el fin de poder realizar estudios comparativos.

En conclusión, se debe seguir ahondando en el estudio de esta temática, sobre todo en áreas psicosociales que permitan aportar datos y estrategias de actuación a las personas en su proceso de envejecimiento, así como a familiares y profesionales, mejorando la atención de manera integral y pudiendo realizar acciones a nivel preventivo, ya que un mayor entendimiento sobre las condiciones que rodean al proceso de envejecimiento de las personas con PC repercutirá en una mejora de su calidad de vida. 


\section{Referencias bibliográficas}

Alcedo, M. Á. et al. (2017): "Personas con discapacidad intelectual que envejecen: evaluación de necesidades percibidas". International Journal of Clinical and Health Psychology, 17 (1): 38-45.

Andersson, C. y Mattsson, E. (2001): "Adults with cerebral palsy: a survey describing problems, needs, and resources, with special emphasis on locomotion”. Developmental Medicine and Child Neurology, 43 (2): 76-82.

Badía-Corbella, M. (2007): "Tendencias actuales de investigación ante el nuevo concepto de parálisis cerebral”. Siglo Cero: Revista Española sobre Discapacidad Intelectual, 38 (3): 25-38.

Badía-Corbella, M. et al. (2013): "Calidad de vida en los pacientes con parálisis cerebral en proceso de envejecimiento”. Rehabilitación, 47 (4): 194-199. doi:10.1016/j.rh.2013.02.002.

Balandin, S. et al. (2006): "Assessing the loneliness of older people with cerebral palsy". Disability and Rehabilitation, 28 (8): 469-479. doi:10.1080/09638280500211759.

Balandin, S. et al. (2009): "Understanding mealtime changes for adults with cerebral palsy and the implications for support services". Journal of Intellectual \& Developmental Disability, 34 (3): 197-206. doi:10.1080/13668250903074489.

Ballin, L. y Balandin, S. (2007): "An exploration of loneliness: Communication and the social networks of older people with cerebral palsy". Journal of Intellectual \& Developmental Disability, 32 (4): 315-326. doi:10.1080/13668250701689256.

Beadle-Brown, J. et al. (2015): "Observing practice leadership in intellectual and developmental disability services”. Journal of Intellectual Disability Research, 59: 1081-1093. doi:10.1111/jir.12208.

Benner, J. L. et al. (2017): "Long-Term Deterioration of Perceived Health and Functioning in Adults with Cerebral Palsy". Archives of Physical Medicine and Rehabilitation, 98 (11): 2196-2205. doi:10.1016/j.apmr.2017.03.013.

Berjano, E. y García, E. (2010): Discapacidad intelectual y envejecimiento: Un problema social del siglo XXI. Madrid: FEAPS.

Bottos, M. et al. (2001): "Functional status of adults with cerebral palsy and implications for treatment of children". Developmental Medicine and Child Neurology, 43 (08): 516-528. doi:10.1017/s0012162201000950.

Brunton, L. K. y Rice, C. L. (2012): "Fatigue in cerebral palsy: A critical review". Developmental Neurorehabilitation, 15 (1): 54-62. doi:10.3109/17518423.2011.629633.

Carmeli, E. et al. (2004): "A comparison between older persons with Down syndrome and a control group: clinical characteristics, functional status and sensorimotor function". Down's syndrome, research and practice: the journal of the Sarah Duffen Centre, 9 (1): 17-24 (en línea). <https://assets.cdn.down-syndrome.org/pubs/a/reports-282.pdf?_ga=2.200546729.625303037.1563216785-2064796747.1563216785>, acceso 22 de noviembre de 2019.

Cans, C. et al. (2000): "Surveillance of cerebral palsy in Europe: a collaboration of cerebral palsy surveys and registers". Developmental medicine and child neurology, 42 (12): 816-824. https://doi.org/10.1017/ S0012162200001511.

Chadwick, D. D. y Jolliffe, J. (2009): "A descriptive investigation of dysphagia in adults with intellectual disabilities". Journal of Intellectual Disability Research, 53 (1): 29-43. doi:10.1111/j.1365-2788.2008.01115.x. 
Cleaver, S. et al. (2008): "Relationship Between Mobility Limitations and the Places Where Older Adults with InteIlectual Disabilities Live”. Journal of Policy and Practice in Intellectual Disabilities, 5 (4): 253-258. doi:10.1111/ j.1741-1130.2008.00186.x.

Coppus, A. M. W. (2013): "People with intellectual disability: What do we know about adulthood and life expectancy?". Developmental Disabilities Research Reviews, 18 (1): 6-16. doi:10.1002/ddrr.1123.

Coyle, C. E. y Mutchler, J. E. (2017): "Aging with Disability: Advancement of a Cross-Disciplinary Research Network". Research on Aging, 39 (6): 683-692.

Coyle, C. et al. (2016): "The role of aging and disability resource centers in serving adults aging with intellectual disabilities and their families: findings from seven states". Journal of Aging and Social Policy, 28 (1): 1-14.

Dark, L. J. et al. (2016): "Communication changes experienced by adults with cerebral palsy as they age". International Journal of Speech-Language Pathology, 18 (6): 521-532. doi:10.3109/17549507.2016.1143976.

Del Barrio, J. S. et al. (2006): "Retos del envejecimiento de las personas con discapacidad intelectual". Revista INFAD de Psicología, 1 (2): 47-56. doi.org/10.17060/ijodaep.2016.n2.v1.559.

Fernández-Ríos, L. y Buela-Casal, G. (2009): "Standards for the preparation and writing of Psychology review articles". International Journal of Clinical and Health Psychology, 9 (2): 329-344.

Frisch, D. y Msall, M. E. (2013): "Health, functioning, and participation of adolescents and adults with cerebral palsy: A review of outcomes research". Developmental Disabilities Research Reviews, 18 (1): 84-94. doi:10.1002/ ddrr.1131.

Galambos, N. L. et al. (2007): "Subjective Age in the Transition to Adulthood for Persons with and without Motor Disabilities". Journal of Youth and Adolescence, 36 (6): 825-834. doi:10.1007/s10964-007-9190-6.

González-Alonso, M. Y. et al. (2017): "Envejecimiento en parálisis cerebral, un reto en investigación e innovación: revisión sistemática”. Universitas Psychologica, 16 (3): 1-15. doi.org/10.11144/Javeriana.upsy16-3.epcr.

Goodwin, D. L. y Compton, S. G. (2004): "Physical Activity Experiences of Women Aging with Disabilities". Adapted Physical Activity Quarterly, 21 (2): 122-138. doi:10.1123/apaq.21.2.122.

Haak, P. et al. (2009): "Cerebral palsy and aging". Developmental Medicine \& Child Neurology, 51:16-23. doi:10.1111/j.1469-8749.2009.03428.x.

Haddad, A. et al. (2007): "A pharmacist's introduction to the effects of aging on patients with cerebral palsy". The Consultant Pharmacist ${ }^{\circledR}, 22$ (8): 669-674. doi.org/10.4140/tcp.n.2007.669.

Heller, T. et al. (2002): “Determinants of exercise in adults with cerebral palsy”. Public Health Nursing, 19 (3): $223-$ 231. doi.org/10.1046/j.0737-1209.2002.19311.x.

Hemsley, B. et al. (2007a): "Family Caregivers Discuss Roles and Needs in Supporting Adults with Cerebral Palsy and Complex Communication Needs in the Hospital Setting". Journal of Developmental and Physical Disabilities, 20 (3): 257-274. doi:10.1007/s10882-007-9095-z.

Hemsley, B. et al. (2007b): “Older Unpaid Carers' Experiences Supporting Adults with Cerebral Palsy and Complex Communication Needs in Hospital”. Journal of Developmental and Physical Disabilities, 19 (2): 115-124. doi:10.1007/s10882-007-9040-1.

Horsman, M. et al. (2010): "Growing Older With Cerebral Palsy". Pediatric Physical Therapy, 22 (3): $296-303$. doi:10.1097/pep.0b013e3181eabc0f. 
Horstmann, H. M. et al. (2009): "Orthopaedic issues in the musculoskeletal care of adults with cerebral palsy". Developmental Medicine \& Child Neurology, 51: 99-105. doi.org/10.1111/j.1469-8749.2009.03417.x.

Instituto Nacional de Estadística (INE) (2008). Encuesta sobre discapacidad, autonomía personal y situaciones de dependencia (en línea). <http://www.ine.es/dyngs/INEbase/es/operacion.htm?c=Estadistica_C\&cid=1254736 176782\&menu=resultados\&idp=1254735573175 >, acceso el 10 de junio de 2018.

Jahoda, A. et al. (2015): "A feasibility study of behavioural activation for depressive symptoms in adults with intellectual disabilities". Journal of Intellectual Disability Research, 59: 1010-1021. doi:10.1111/jir.12175.

Jones, G. C. (2009): "Aging with cerebral palsy and other disabilities: personal reflections and recommendations". Developmental Medicine \& Child Neurology, 51: 12-15.

Judge, J. et al. (2010): "Activity, aging, and retirement: The views of a group of Scottish people with intellectual disabilities". Journal of Policy and Practice in Intellectual Disabilities, 7: 295-301. doi:10.1177/0164027504271349.

Klingbeil, H. et al. (2004): "Aging with a disability". Archives of physical medicine and rehabilitation, 85: 68-73. doi:10.1016/j.apmr.2004.03.014.

Lifshitz, H. et al. (2008): "Health status and ADL functioning of older persons with intellectual disability: Community residence versus residential care centers". Research in Developmental Disabilities, 29 (4): $301-315$. doi:10.1016/j.ridd.2007.06.005.

Low Choy, N. et al. (2003): "The efficacy of a work-station intervention programme to improve functional ability and flexibility in ageing clients with Cerebral Palsy: a pilot study". Disability and Rehabilitation, 25 (21): 1201-1207. doi:10.1080/09638280310001599998.

Martin, L. et al. (2017): "The Power of Population Health Data on Aging and Intellectual and Developmental Disabilities: Reactions of Knowledge Users". Journal of Policy and Practice in Intellectual Disabilities, 14: 268-278. doi.org/10.1177/1744629511413506.

McCallion, P. et al. (2019): "Epidemiological Issues in Intellectual Disability and Aging Research", en Prasher V. y Janicki M. (eds.): Physical Health of Adults with Intellectual and Developmental Disabilities . Birmingham: Springer. doi.org/10.1007/978-3-319-90083-4_5.

McPhee, P. G. et al. (2016): "Fatigue and its relationship with physical activity, age, and body composition in adults with cerebral palsy”. Developmental Medicine \& Child Neurology, 59 (4): 367-373. doi:10.1111/dmcn.13306.

Michelsen, S. I. et al. (2006): "Social integration of adults with cerebral palsy". Developmental Medicine \& Child Neurology, 48 (08): 643. doi:10.1017/s0012162206001368.

Mitchell, J. M. et al. (2006): "The Effects of Aging on Employment of People With and Without Disabilities". Rehabilitation Counseling Bulletin, 49 (3): 157-165. doi:10.1177/00343552060490030301.

Moll, L. R. y Cott, C. A. (2012): "The paradox of normalization through rehabilitation: growing up and growing older with cerebral palsy”. Disability and Rehabilitation, 35 (15): 1276-1283. doi:10.3109/09638288.2012.726689.

Morgan, P. et al. (2014): "Health service experiences to address mobility decline in ambulant adults ageing with cerebral palsy". Journal of Intellectual \& Developmental Disability, 39 (3): 282-289. doi:10.3109/13668250.20 14.927841.

Morgan, P. y McGinley, J. (2014): "Gait function and decline in adults with cerebral palsy: a systematic review". Disability and Rehabilitation, 36 (1): 1-9. doi:10.3109/09638288.2013.775359. 
Morgan, P. et al. (2015): "Perceived cause, environmental factors, and consequences of falls in adults with cerebral palsy: a preliminary mixed methods study". Rehabilitation research and practice, 2015. doi. org/10.1155/2015/196395.

Moro, T. T. et al. (2017): "Agency, social and healthcare supports for adults with intellectual disability at the end of life in out-of-home, non-institutional community residences in Western nations: A literature review". Journal of Applied Research in Intellectual Disabilities. 30 (6):1045-1056. doi.org/10.1111/jar.12374.

Moher, D. et al. (2009): "Preferred Reporting Items for Systematic Reviews and MetaAnalyses: The PRISMA Statement”. PLoS Medicine, 6: e1000097. doi:10.1371/journal.pmed1000097.

Mudge, S. et al. (2016): "Ageing with cerebral palsy; what are the health experiences of adults with cerebral palsy? A qualitative study”. BMJ Open, 6 (10): e012551. doi:10.1136/bmjopen-2016-012551.

Ng, N. et al. (2015): "Prevalence of older people with intellectual disability in Sweden: a spatial epidemiological analysis". Journal of Intellectual Disability Research, 59: 1155-1167. doi:10.1111/jir.12219.

OMS (2001): Clasificación Internacional del Funcionamiento de la Discapacidad y de la Salud (CIF). Madrid: IMSERSO.

Perestelo-Pérez, L. (2013): "Standards on how to develop and report systematic reviews in Psychology and Health". International Journal of Clinical and Health Psychology, 13 (1): 49-57. doi.org/10.1016/S1697-2600(13)700073 Get.

Peterson, M. D. et al. (2013): "Chronic disease risk among adults with cerebral palsy: the role of premature sarcopenia, obesity and sedentary behaviour”. Obesity Reviews, 14 (2): 171-182. doi:10.1111/j.1467789x.2012.01052.x.

Peterson, M. D. et al. (2012): "Secondary muscle pathology and metabolic dysregulation in adults with cerebral palsy". American Journal of Physiology-Endocrinology and Metabolism, 303 (9): E1085-E1093. doi:10.1152/ ajpendo.00338.2012.

Petretto, D. R. et al. (2019): "Aging and disability: the need of a bridge to promote wellbeing". Gerontology \& Geriatric Medicine, 4 (5): 1-6. doi: 10.19080 / OAJGGM.2019.04.555648.

Platt, M. J. (2016): “Counting cases of cerebral palsy”. Developmental Medicine \& Child Neurology, 59 (3): 243. doi:10.1111/dmen.13304.

Pueyo-Benito, R. y Vendrell-Gómez, P. (2002): “Neuropsicología de la Parálisis Cerebral”. Revista de Neurología, 34: 1080-1087.

Robaina, G. R. et al. (2007): "Definición y clasificación de la parálisis cerebral: ¿un problema ya resuelto?”. Revista de Neurología, 45 (2): 110-117. doi.org/10.33588/rn.4502.2006595.

Rosenbaum, P. et al. (2007): "A report: the definition and classification of cerebral palsy". Dev Med Child Neurol, Suppl. 109: 8-14.

Sandström, K. et al. (2009): "Prerequisites for carrying out physiotherapy and physical activity - experiences from adults with cerebral palsy”. Disability and Rehabilitation, 31 (3): 161-169. doi:10.1080/09638280701850934.

Schepens, H. R. et al. (2017): "What Indicates and Determines Quality of Life for Elderly People with Intellectual Disabilities?". Journal of Mental Health Research in Intellectual Disabilities, 10: 46-46. doi.org/10.1111/jar.12559.

Shortland, A. (2009): "Muscle deficits in cerebral palsy and early loss of mobility: can we learn something from our elders?". Developmental Medicine \& Child Neurology, 51: 59-63. doi.org/10.1111/j.1469-8749.2009.03434.x. 
Svien, L. R. et al. (2008): "Issues in aging with cerebral palsy". Topics in Geriatric Rehabilitation, 24 (1): $26-40$. doi:10.1111/ j.1469-8749.2009.03428.x.

Strydom, A. et al. (2019): "Physical Health and Clinical Phenotypes", en Prasher, V. y Janicki, M. (eds.): Physical Health of Adults with Intellectual and Developmental Disabilities. Birmingham: Springer. doi.org/10.1007/9783-319-90083-4_5.

Tarsuslu, T. y Livanelioglu, A. (2010): "Relationship between quality of life and functional status of young adults and adults with cerebral palsy”. Disability and Rehabilitation, 32 (20): 1658-1665. doi:10.3109/09638281003649904.

Taylor, N. F. et al. (2004): "Adults with cerebral palsy benefit from participating in a strength training programme at a community gymnasium". Disability and Rehabilitation, 26 (19): 1128-1134. doi:10.1080/0963828041000171 2387.

Thorpe, D. (2009): "The role of fitness in health and disease: status of adults with cerebral palsy". Developmental Medicine \& Child Neurology, 51: 52-58. doi.org/10.1111/j.1469-8749.2009.03433.x.

Tosi, L. L et al. (2009): "Adults with cerebral palsy: a workshop to define the challenges of treating and preventing secondary musculoskeletal and neuromuscular complications in this rapidly growing population". Developmental Medicine \& Child Neurology, 51: 2-11. doi:10.1111/j.1469-8749.2009.03462.x.

Turk, M. A. (2009): "Health, mortality, and wellness issues in adults with cerebral palsy". Developmental Medicine \& Child Neurology, 51: 24-29. doi.org/10.1111/j.1469-8749.2009.03429.x.

Turk, M. A. et al. (2001): "The health of women with cerebral palsy". Physical medicine and rehabilitation clinics of North America, 12 (1): 153-168. doi.org/10.1016/j.apmr.2004.03.014.

Verdugo, M. A. y Navas, P. (2017): Todos somos todos: derechos y calidad de vida de las personas con discapacidad intelectual y mayores necesidades de apoyos. Madrid: Real Patronato sobre Discapacidad. 